\title{
A Portable Autonomic Nervous Activity Monitor
}

\author{
Ping Shi ${ }^{1,2,3^{*}}$ and Yankun $\mathrm{Xu}^{1}$ \\ ${ }^{1}$ Institute of rehabilitation engineering and technology, University of Shanghai for Science and Technology, Shanghai, P. R. China \\ ${ }^{2}$ Shanghai Engineering Research Center of Assistive Devices, Shanghai, P. R. China \\ ${ }^{3}$ Key Laboratory of Neural-functional Information and Rehabilitation Engineering of the Ministry of Civil Affairs, Shanghai, \\ P. R .China \\ *Corresponding author
}

\begin{abstract}
A portable system has been designed to enable monitoring of autonomic nervous Activity. The present invention discloses a portable, simple, and cost-effective electronic device containing hardware and software that permit real-time monitoring of photoplethysmography (PPG) waveform. HRV parameters including time- and frequency-domain analysis extract from PPG signal were used to reflect the changes in the autonomic nervous system.
\end{abstract}

Keywords-portable monitor; autonomic nervous activity; photoplethysmography; heart rate variability

\section{INTRODUCTION}

A variety of perioperative factors such as anxiety, pain and surgical trauma are known to cause an imbalance of the autonomous nervous system (ANS). Time- and frequencydomain analysis of heart rate variability (HRV) is a noninvasive tool that can be used to investigate ANS activity [1]. There are two primary technologies for measuring heart rate metrics: electrocardiography (ECG) [2] and photoplethysmography (PPG) [3]. ECG measures the biopotential generated by electrical signals that control the expansion and contraction of heart chambers, while PPG uses a light-based technology to sense the rate of blood flow as controlled by the heart's pumping action. The electro-optic technique of measuring the cardiovascular pulse wave known as photoplethysmography (PPG) is clinically utilized for noninvasive characterization of physiological components by dynamic monitoring of tissue optical absorption. There has been a resurgence of interest in this technique in recent years, driven by the demand for a low cost, compact, simple and portable technology for the primary care and community-based clinical settings and the advancement of computer-based pulse wave analysis techniques. PPG has been proposed as an alternative for ECG in HRV analysis to overcome some difficulties in measurement of ECG [4-6]. Therefore, HRV based on PPG is a widely acceptable technology used in portable devices as a modern optical wrist-worn heart rate monitors. The present invention discloses a portable, simple, and cost-effective electronic device containing hardware and software that permits real-time monitoring of a pulsatile blood volume waveform obtained through use of a PPG probe. The HRV parameters were extracted from PPG waveform to assess the autonomic nervous activity.

\section{SYSTEM DESIGN}

\section{A. Structure of the System}

A block diagram of the portable autonomic activity monitor is shown in Figure I. A photoelectric sensor is used to acquire primitive physiological signals. In order to extract the useful signals, signal conditioning is designed to process the raw signal by amplification and filtering. Because of the characteristic of computer which cannot process the analog signal, an onboard analog to digital conversion is used. The processed signal is stored in the microcontrollers. Then the signal is transmitted to Android via bluetooth low energy (BLE), where the useful parameters are acquired and displayed. In the next Section, the function of each component will be briefly discussed.

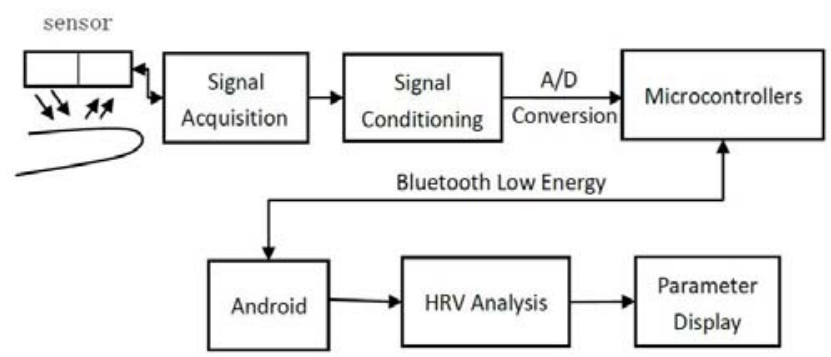

FIGURE I. BLOCK DIAGRAM OF THE SYSTEM

\section{B. Hardware Design}

1) PPG signal acquisition module: The PPG signals, as shown in Figure II, are composed of two components: a DC component, which is a relatively constant voltage, but changes in magnitude depending on the nature of the tissue through which the light passes, and an AC or pulsatile component synchronous with HR and is superimposed onto a large quasiDC component that relates to the tissues and to the average blood volume. The AC pulse shapes are indicative of vessel compliance and cardiac performance and the amplitude is usually 1 to $2 \%$ of the DC value. 


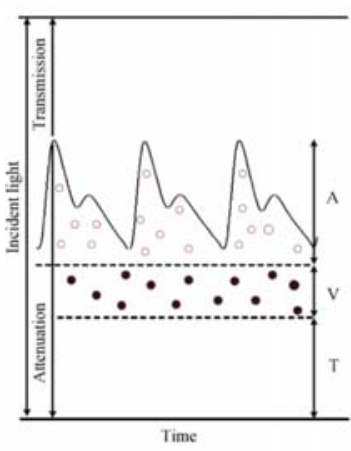

(a)

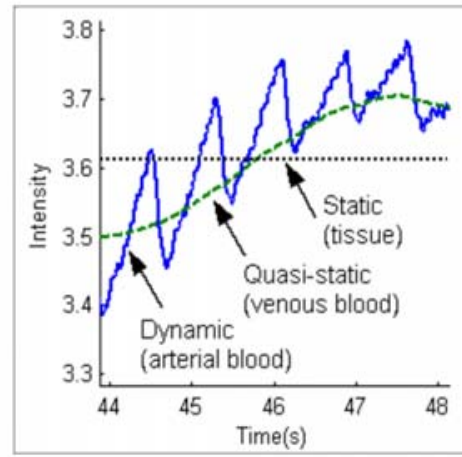

(b)
FIGURE II. PPG SIGNAL. (A) SCHEMATIC OF THE PULSATILE PHOTOELECTRIC OUTPUT. THE ATTENUATION OF THE INCIDENT LIGHT IS CAUSED BY A---ARTERIAL BLOOD, V--VENOUS BLOOD, T---TISSUES. (B) COMPONENTS OF A RAW PPG SIGNAL

Model APDS-9008 (Avago Co, Ltd, American), which can accept light with a wavelength less than $565 \mathrm{~nm}$, is used in this system. In order to improve the signal-to-noise ratio of the original pulse signal and increase the amplitude of the output signal, AM2520 (Kingbright Electronic Co, Ltd, Taiwan, China) which can emit green light with a wavelength of $515 \mathrm{~nm}$ is used. The raw PPG signal is shown in Figure III.

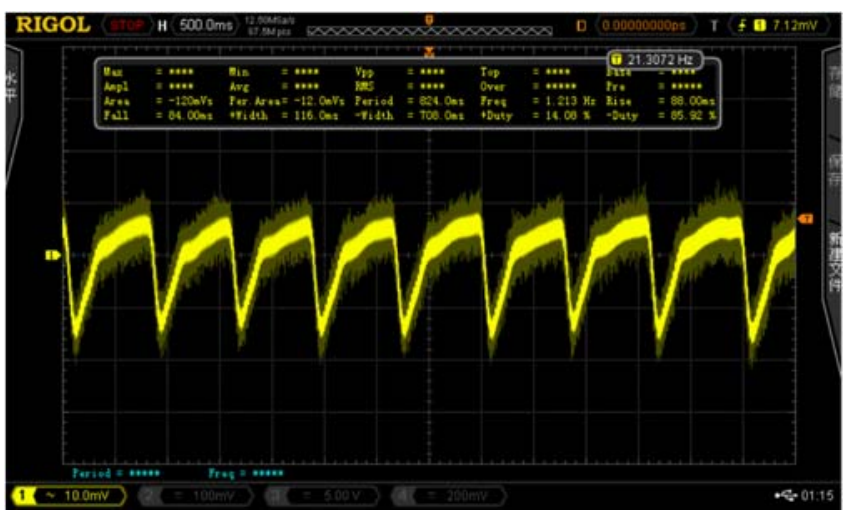

FIGURE III. RAW PPG SIGNAL

2) PPG signal amplifier and filter: Normally, the heart rate of adults is 60 to 120 beats per minute $(1 \sim 2 \mathrm{~Hz})$. In order to extract useful information, a band-pass filter which own a bandwidth of $0.07 \sim 3.38 \mathrm{~Hz}$ is designed to filter the raw signal. The filtered signal is typically a microvolt level, which is too weak for the further process. Therefore, an operational amplifier based on MCP6001 (Microchip Co, Ltd, Arizona, American) is designed, which can amplify the signal 330 times. Then the amplified signal is transmitted to microcontroller (MCU) for further processing. A block diagram of signal conditioning is showed in Figure IV. The signal processing circuit is shown in Figure V. The signal after amplifying and filtering is shown in Figure VI.

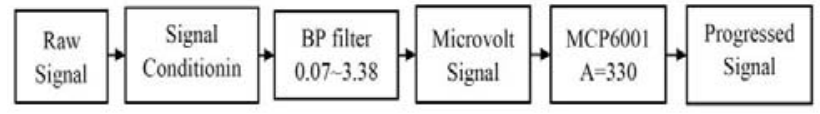

FIGURE IV. BLOCK DIAGRAM OF SIGNAL CONDITIONING

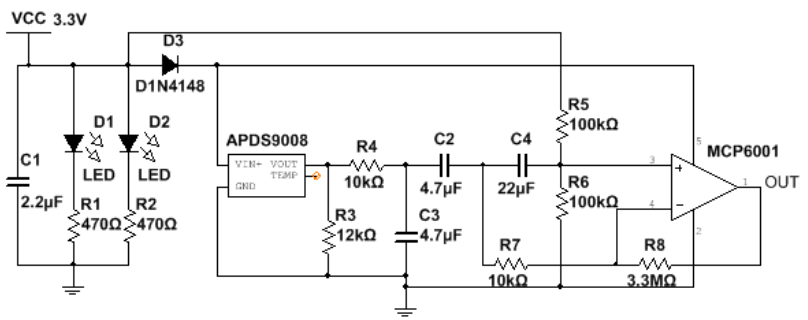

FIGURE V. THE SIGNAL PROCESSING CIRCUIT

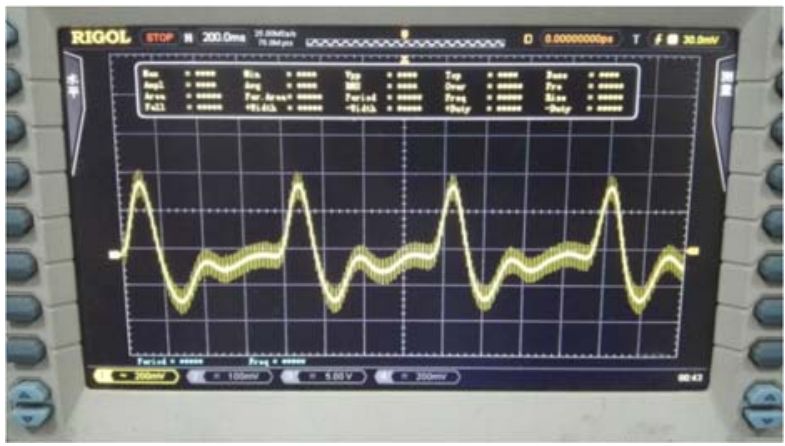

FIGURE VI. THE SIGNAL AFTER AMPLIFIER AND FILTER

3) Microcontroller: STM32L152 (STMicroelectronics Co,Ltd, Geneva, Switzerland) was selected as microcontroller of the system, which is a low-power chip with built-in 12-bit ADC. The operating frequency of STM32L152 is 32MHz, and has an ARM Cortex-M3 core.

4) Power supply circuit: The system is powered by lithium batteries with power supply voltage of $3.7 \mathrm{~V}$. However, the operating voltage of sensor and microcontroller is $3.3 \mathrm{~V}$, and the system is sensitive to chip size. Therefore, MCP1700T was selected as a step-down chip. The power supply circuit is shown in Figure VII.

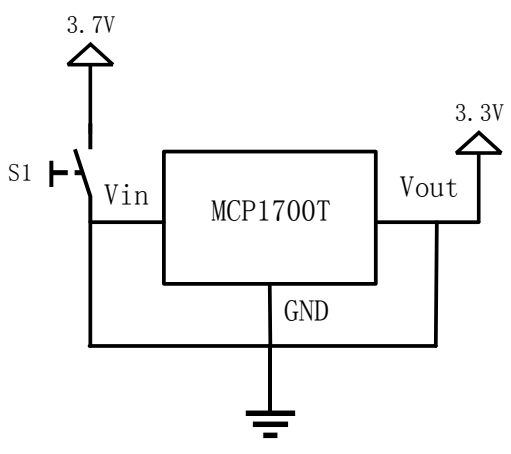

FIGURE VII. POWER SUPPLY CIRCUIT

5) Bluetooth module: HJ-580 (HongJia Co, China) is used to wirelessly transmit the PPG signal to the Android 
smartphone (Figure VIII). It is chosen because of its small size of around $5 \times 6.2 \mathrm{~mm}$ and its characteristics. The average operating current of the bluetooth module at full speed is only $1.5 \mathrm{~mA}$, and the maximum power consumption is only $300 \mathrm{uA}$.

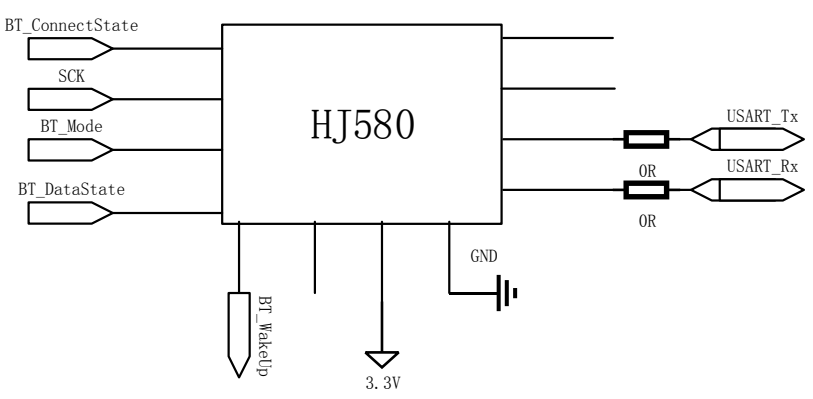

FIGURE VIII. BLUETOOTH MODULE

\section{Program Design}

Once the hardware is designed, how to drive it also be considered. An overall design block diagram of the program is shown in Figure IX. The program design can be divided into two parts: signal recording and signal progressing.

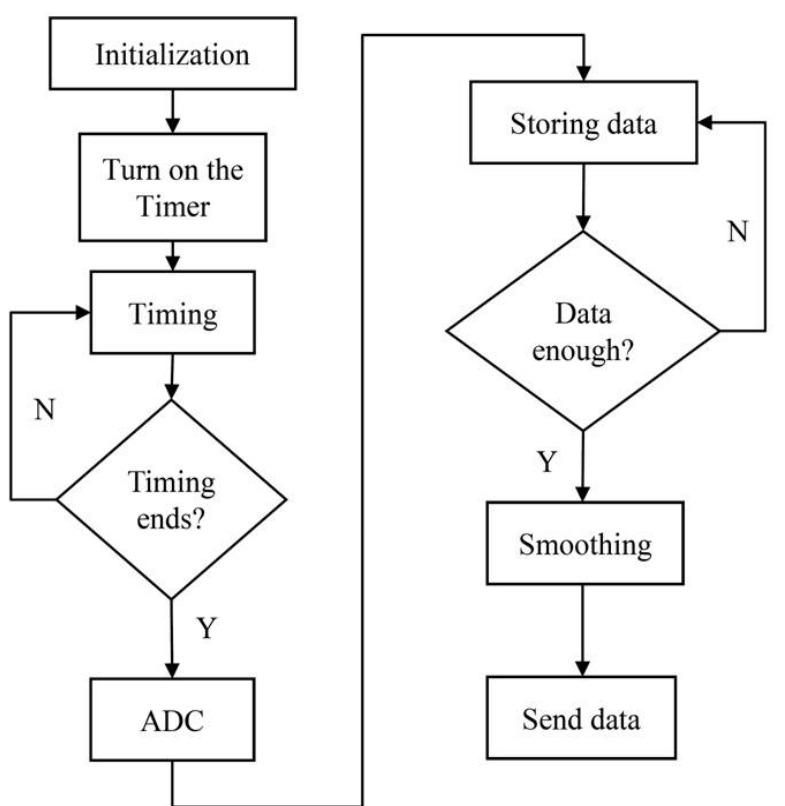

FIGURE IX. OVERALL DESIGN BLOCK DIAGRAM

1) Signal recording: In order to record signal, timer, ADC and USART (universal synchronous asynchronous receiver transmitter) of microcontroller must be opened and configured. In this system, onboard timer (timer2) was selected and APB1 was chosen as a clock. In the clock configuration of the ADC, analog part of the clock should use a separated clock, which is shown in Figure X. Because the importance of accurate data is higher than the transmission speed, the USART is set to asynchronous communication mode.

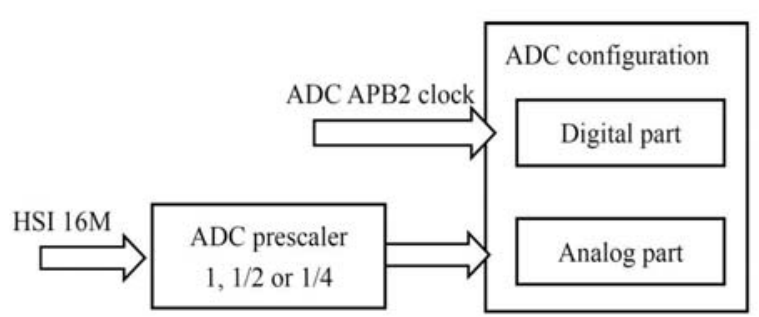

FIGURE X. CLOCK CONFIGURATION OF THE ADC

2) Signal progressing: The raw signal has been filtered and amplified by hardware circuit. As it's shown in Figure $\mathrm{XI}(\mathrm{a})$, a lot of glitches were also found in the waveform, which carried caused errors while calculating the heart rate. The glitch can be removed by smoothing. The quadratic smoothing algorithm with five-point approximation was selected to smoothing the waveform after comparison. As shown in Figure XI(b), the waveform is smoother than that before smoothing.

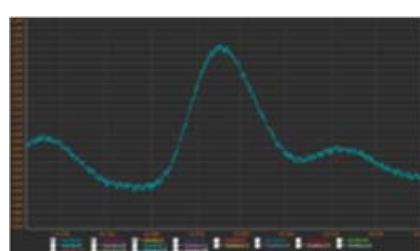

(a)

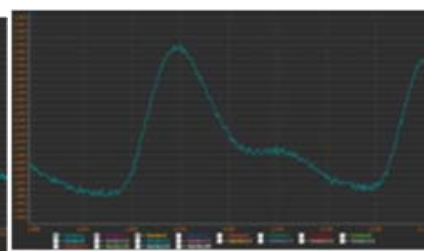

(b)
FIGURE XI. SIGNAL PROGRESSING. (A) BEFORE SMOOTHING; (B) AFTER SMOOTHING.

\section{Android Application Design}

In order to display the PPG signal and receive the information in smartphone devices, an Android application was developed, and which was designed via Android Studio (Google Co, Ltd, California, American).

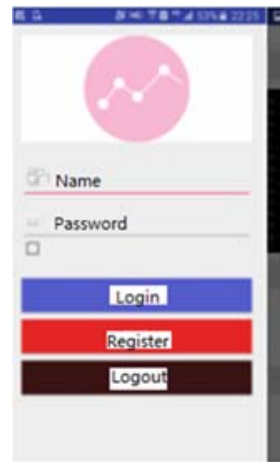

(a)

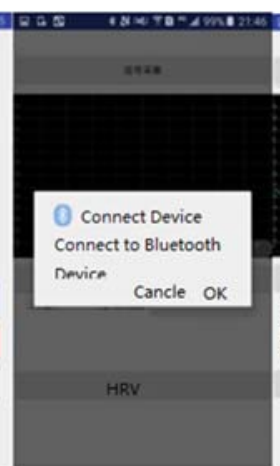

(b)

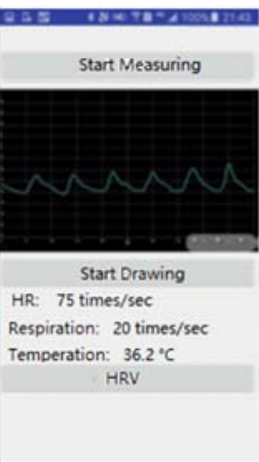

(c)
FIGURE XII. INTERFACE DESIGN. (A) LOGIN INTERFACE; (B) CONNECT TO THE DEVICE; (C) MAIN INTERFACE

1) Interface design: Interface design contains login interface and main interface, as shown in Figure VIII. As the first interface after user open the application, the login interface would confirm the user's identity. As shown in Figure XII, one can be identified as a new or old user by "Login" or "Register", which means whether the system loads data from a sever. Once 
the user logs in the system, a dialog will appear to confirm that the user is connected to the device via bluetooth. Then, the smartphone will receive data from device, and show the waveform in the screen. HRV analysis will be done after clicking the button.

2) HRV analysis: Table I shows the descriptions and definitions of HRV measurement terminology. Through HRV analysis, we obtain the variety of the activities of sympathetic and parasympathetic nerves of the person, i.e. the situation of ANS.

TABLE I. TIME- AND FREQUENCY-DOMAIN MEASURES OF HRV

\begin{tabular}{|c|c|c|}
\hline Measures & Units & Description \\
\hline$H R$ & bmp & Heart rate \\
\hline SDNN & $\mathrm{ms}$ & Standard deviation of all NN intervals. \\
\hline$S D S D$ & $\mathrm{~ms}$ & $\begin{array}{l}\text { Standard deviation of differences between } \\
\text { adjacent NN intervals. }\end{array}$ \\
\hline RMSSD & ms & $\begin{array}{l}\text { The square root of the mean of the sum of the } \\
\text { squares of differences between adjacent NN } \\
\text { intervals. }\end{array}$ \\
\hline $\begin{array}{l}\text { NN50 } \\
\text { count }\end{array}$ & & $\begin{array}{l}\text { Number of pairs of adjacent NN intervals } \\
\text { differing by more than } 50 \mathrm{~ms} \text { in the entire } \\
\text { recording. }\end{array}$ \\
\hline pNN50 & $\%$ & $\begin{array}{l}\text { NN50 count divided by the total number of all } \\
\text { NN intervals. }\end{array}$ \\
\hline$H F$ & $\mathrm{~ms}^{2}$ & $\begin{array}{l}\text { Power in high frequency range in frequency } \\
\text { domain measures. }\end{array}$ \\
\hline$L F$ & $\mathrm{~ms}^{2}$ & $\begin{array}{l}\text { Power in low frequency range in frequency } \\
\text { domain measures. }\end{array}$ \\
\hline
\end{tabular}

\section{EXPERIMENT AND RESULT}

Three healthy subjects participated in the experiment. 5 minute signal from the present portable system based on PPG technology was collected, while a stranded ECG signal was recorded synchronous from a scientific research instrument. Some important time domain HRV parameters were calculated for the comparisons between PPG-HRV and ECG-HRV. In table II, the results show that the parameters from the present portable autonomic nervous activity monitor were acceptable and available.

TABLE II. THE COMPARISION OF PPG-HRV AND ECG-HRV

\begin{tabular}{|c|c|c|c|c|}
\hline \multirow{2}{*}{} & Subjects & S1 & S2 & S3 \\
\cline { 2 - 5 } & Age (yrs) & 51 & 47 & 41 \\
\cline { 2 - 5 } & $\mathrm{M} / \mathrm{F}$ & $\mathrm{M}$ & $\mathrm{F}$ & $\mathrm{M}$ \\
\hline \multirow{2}{*}{ SDNN(ms) } & PPG & 38.7 & 35 & 45 \\
\cline { 2 - 5 } & ECG & 36.9 & 33.5 & 43.7 \\
\hline \multirow{2}{*}{ SDSD(ms) } & PPG & 11.1 & 14.5 & 9.4 \\
\cline { 2 - 5 } & ECG & 13.5 & 16.7 & 12.5 \\
\hline \multirow{2}{*}{ RMSSD(ms) } & PPG & 17.4 & 26 & 13.9 \\
\cline { 2 - 5 } & ECG & 19.6 & 23.4 & 16.4 \\
\hline \multirow{2}{*}{ NN50 } & PPG & 47 & 69 & 39 \\
\cline { 2 - 5 } & ECG & 43 & 59 & 35 \\
\hline
\end{tabular}

In the future development, we would test more subjects and compare our result with the standard Holter ECG medical equipment for accuracy evaluation. On the other hand, we would like to extend the program in Android, so that it can transmit the PPG data to the Internet via 4G networks.

\section{ACKNOWLEDGEMENTS}

This work was supported by Shanghai Engineering Research Center of Assistive Devices (Grand No. 15DZ2251700).

\section{REFERENCES}

[1] U. Rajendra Acharya, K. Paul Joseph, , N.Kannathal, , C. M. Lim, and Suri, J. S., "Heart rate variability: a review," Med Biol Eng Comput, vol. 44, pp. 1031-51, Dec 2006.

[2] S. Iravanian, A. Arshad, , and J. S. Steinberg, "Role of electrophysiologic studies, signal-averaged electrocardiography, heart rate variability, T-wave alternans, and loop recorders for risk stratification of ventricular arrhythmias," Am J Geriatr Cardiol, vol. 14, pp. 16-9, Jan-Feb 2005.

[3] J. Allen, "Photoplethysmography and its application in clinical physiological measurement," Physiol Meas, vol. 28, pp. R1-39, Mar 2007.

[4] V. Jeyhani, S. Mahdiani, , M. Peltokangas, , and A. Vehkaoja, "Comparison of HRV parameters derived from photoplethysmography and electrocardiography signals," Conf Proc IEEE Eng Med Biol Soc, vol. 2015, pp. 5952-5, 2015.

[5] S. Lu, H. Zhao, K. Ju, K. Shin, M. Lee, K. Shelley, and K. H. Chon, "Can photoplethysmography variability serve as an alternative approach to obtain heart rate variability information?," Journal of clinical monitoring and computing, vol. 22, pp. 23-9, 2008.

[6] P. Shi, S. J. Hu, and Y. S. Zhu, "A Preliminary Attempt to Understand Compatibility of Photoplethysmographic Pulse Rate Variability with Electrocardiogramic Heart Rate Variability," Journal of Medical and Biological Engineering, vol. 28, pp. 173-180, 2008 